\title{
Docencia de Bioestadística en Medicina con software gratuito jamovi: una ventana de oportunidad.
}

\section{Biostatistics in Medicine teaching with free software jamovi: a window of opportunity.}

\author{
Javier Santabárbara ${ }^{1 *}$, Isabel Lasheras ${ }^{1,2}$ \\ 1 Departamento de Medicina Preventiva y Salud Pública. Área de Bioestadística. Facultad de \\ Medicina, Universidad de Zaragoza, Zaragoza, España; jsantabarbara@unizar.es, \\ https://orcid.org/0000-0002-7297-6104 \\ 2 738605@unizar.es \\ * Correspondencia: jsantabarbara@unizar.es
}

Recibido: 3 de abril de 2020; Aceptado: 3 de mayo de 2020; Publicado: 3 de mayo de 2020

La enseñanza de la Estadística, cuya ubicuidad en los programas académicos responde a la capacidad de esta ciencia de estructurar la lógica del estudiante y brindar elementos para el manejo de la información y la investigación (1), cobra especial relevancia en el ámbito de la medicina, donde el profesional médico deberá valorar la relación riesgo-beneficio que comporta toda intervención diagnóstica o terapéutica que se le practique al paciente, valiéndose de la estadística para tomar decisiones en situaciones de incertidumbre (2).

En los últimos años, en las universidades españolas, se han utilizado programas como SPSS para la docencia de la Estadística en Medicina. Este paquete estadístico permite realizar una amplia gama de análisis y su inclusión en el programa docente responde a su extendido uso hospitalario. Sin embargo, posee la seria limitación de ser software propietario, con un coste prohibitivo para el uso individual del alumnado. Tampoco permiten acceder al código fuente, por lo que no es fácilmente adaptable a las necesidades docentes (3).

El uso de software libre es una herramienta recomendada por el Servicio de Informática de algunas Universidades, por cuanto se considera que fortalece la libertad de cátedra y del alumnado, aporta a la institución independencia e interoperabilidad para el funcionamiento de sus sistemas de información y garantiza el libre acceso de la comunidad universitaria a la información necesaria para llevar a cabo sus actividades (4).

Hasta la fecha, se optaba por el uso alternativo del software libre R (5), ampliamente extendido entre la comunidad científica, pero cuya principal desventaja frente a SPSS es su elevada dificultad de aprendizaje, ya que se trata de un lenguaje de comandos $(1,3)$. Frente a esta opción, ha surgido recientemente jamovi (6), un software de acceso gratuito y multiplataforma (está disponible para Linux, Mac, Windows y ChromeOS) que utiliza el lenguaje de programación de R. Además, proporciona una interfaz amable con el usuario, similar a la de SPSS, afianzándose como una alternativa potente a éste. Adicionalmente, ofrece la posibilidad de visualizar la sintaxis, sirviendo también como puente para usuarios familiarizados con interfaces de menús que deseen iniciarse en el lenguaje de comandos de $\mathrm{R}$.

De este modo, su facilidad de uso lo convierte en una herramienta docente sencilla, al tiempo que la posibilidad de añadir módulos avanzados asegura su utilidad 
futura en el postgrado y, porqué no, en el desempeño investigador de muchos de los estudiantes actuales de Medicina. Actualmente existen ya doce módulos accesibles desde la aplicación $\mathrm{y}$, siendo la trasposición de los paquetes de $\mathrm{R}$ a jamovi $\mathrm{y}$ el desarrollo de funciones "a medida" por el usuario relativamente sencillo, parece haber un futuro muy prometedor para este software. Otra de sus ventajas de cara a su aplicación para la docencia es su reproducibilidad, pues el programa guarda las opciones seleccionadas en los cuadros de diálogo y, además, lo hace junto a los análisis y gráficos en un solo fichero, lo que facilita la colaboración entre autores.

Los docentes de Bioestadística en Medicina nos encontramos ante la oportunidad de enseñar el manejo de jamovi con la intención de que los futuros médicos puedan realizar el tratamiento estadístico de sus investigaciones sin asumir coste alguno y evitando tener que aprender un lenguaje de programación estadístico.

Material suplementario: no hay.

Financiación: No ha habido financiación.

Declaración de conflicto of interés: Los autores declaran no tener ningún conflicto de intereses

\section{Referencias}

1. Bastidas JAO. El R, una herramienta TIC para la enseñanza-aprendizaje de la estadística. 2014. https://www.fcfm.buap.mx/EIEPE2017/memorias-EIEPE2017/extenso/P21.pdf

2. Dawson GF. Interpretación fácil de la bioestadística. La conexión entre la evidencia y las decisiones médicas. Barcelona (España): Elsevier; 2009.

3. Sánchez Alberca A. rkTeaching: un paquete de R para la enseñanza de Estadística. In: Docencia en Estadística Experiencias de Innovación. 2013. p. 167-79. http://metodos.upct.es/jidere/files/libro jidere 13.pdf

4. Consejo de Gobierno de la Universidad de Zaragoza. Declaración Institucional de la Universidad de Zaragoza sobre "Software libre y de fuentes Abiertas." 2007. https://osluz.unizar.es/info/declaracion

5. R Core Team (2019). R: A language and environment for statistical computing. R Foundation for Statistical Computing, Vienna, Austria. https://www.R-project.org/

6. The jamovi project (2020). jamovi (Version 1.2) [Computer Software]. Retrieved from https:// www.jamovi.org

(C) 2020 por los autores. Enviado para su posible publicación en acceso abierto bajo los términos y condiciones de la licencia Creative Commons Attribution (CC BY) (http://creativecommons.org/licenses/by/4.0/). 